Turkish Journal of Geriatrics

DOI: 10.31086/tigeri.2020.184

2020; 23(4): 463-472

- Nergiz HÜSEYINOĞLU1 D

- Hatice KÖSE ÖZLECE ${ }^{2}$ (D)

- Sadık ARDIÇ3 ${ }^{3}$

CORRESPONDANCE

${ }^{1}$ Nergiz Hüseyinoğlu

Aclbadem University, Acibadem Kayseri Hospital Department of Neurology and Sleep Diseases, Kayseri, Turkey

Phone: +905058119172

e-mail: nergizabbas@gmail.com

Received: Jul 24, 2020

Accepted: Nov 24, 2020

1 Aclbadem University, Acibadem Kayseri Hospital Department of Neurology and Sleep Diseases, Kayseri, Turkey

${ }_{2}^{2}$ Acibadem Kayseri Hospital, Depatment of Neurology and Sleep Diseases, Kayseri, Turkey

${ }^{3}$ Koru Ankara Hospital, Department of Chest and Sleep diseases, Ankara, Turkey

\section{COMPARISON OF SLEEP MACRO- AND MICROSTRUCTURES IN ELDERLY AND MIDDLE-AGED MALE PATIENTS WITH SEVERE OBSTRUCTIVE SLEEP APNEA: DOES THE DISEASE ERASE DIFFERENCES?}

\section{Abstract}

Introduction: Age-related changes in sleep attract increased attention from researchers and are studied in the context of micro- and macrostructures. Macrostructures include sleep onset, total sleep time, sleep efficiency, wake time after sleep onset, and non-rapid eye movement and rapid eye movement sleep percentages, latencies and durations. Sleep microstructures refer to a cyclic alternating pattern.The present study aims to investigate how sleep structures in older and middle-aged patients are affected by the widespread disease of obstructive sleep apnea. The authors believe that it provide additional information about sleep disorder pathogenesis.

Materials and Method: Patients who received a diagnosis of severe sleep apnea were divided into two groups: age $\geq 65$ years old and 35-45 years old. Macro-and microstructural data from polysomnographic investigations were collected and compared.

Results: There were no statistically significant differences for total sleep time, sleep efficiency, wake time after sleep onset, rapid eye movement sleep latency and separate percentages of non-rapid eye movement sleep stages N1, N2 and N3. Regarding sleep microstructures, there were no differences between arousal index, cyclic alternating pattern rates, or percentage ratio of phase subtype.

Conclusion: The parameters of the macro- and microstructures in the older group are similar to those of the middle-aged group and are also basically similar to the parameters of previous studies; however, the percentages from phases A1, A2 and $A 3$ are different from most previous works. These considerations indicate the need for future investigation of geriatric sleep in different health and environmental conditions.

Keywords: Sleep; Sleep Stages; Sleep Apnea Syndromes; Aging; Polysomnography 


\section{INTRODUCTION}

Sleep is an integral part of human life and is one of the main factors determining vitality, consciousness, mental functions, homeostasis and other physiological and psychosocial aspects of being human. Sleep is not a stable state, and its architecture dynamics change with age. Generally speaking, sleep in humans consists of cyclic turnover between rapid eye movement (REM) and non-REM (NREM) sleep. NREM sleep consists of light (N1 and N2) and deep (N3 or slow wave) sleep stages. REM sleep represents with a decrease in voluntary muscle tone and rapid eye movement. Aging per se is not the single reason for sleep disruption. The deterioration of architecture and quality of elderly people's sleep are caused by altered homeostatic sleep regulation, lifestyle changes, physical activity and diet properties, comorbidities, chronic pain, medications and senile brain changes.

The sleep structures are evaluated by EEG, divided into 30-second epochs to observe sleep macro- and microstructures. Macrostructures include
NREM and REM sleep percentages, latencies and durations.

The definition of sleep microstructure includes waves which do not belong to the conventional 30 -s scoring epoch $(1,2)$. One of the microstructural phenomenon is the cyclic alternating pattern (CAP), which is defined as aperiodic activity during NREM sleep and reflects the brain's efforts in regulating the physiological structure of sleep $(1,3)$. CAP is defined as repetitive spontaneous EEG patterns (arousal period/Phase A) lasting 10-60 s and subsequent return to background activity (quiet period/Phase B) (Figure 1) (1). The amount of CAP increases during sleep disruption, such as acoustic stimulation, insomnia, pain, sleep apnea, periodic limb movement or depression. Conversely, drug administration, narcolepsy or degenerative diseases reduce the CAP rate (4). CAP provides additional information about NREM sleep and may shed light on sleep disorder pathogenesis (3).

According to many studies, slow wave sleep (SWS), REM sleep, SE and TST decrease, but per-

Figure 1. Cyclic alternating pattern: arousal period (phase A), background period (phase B)

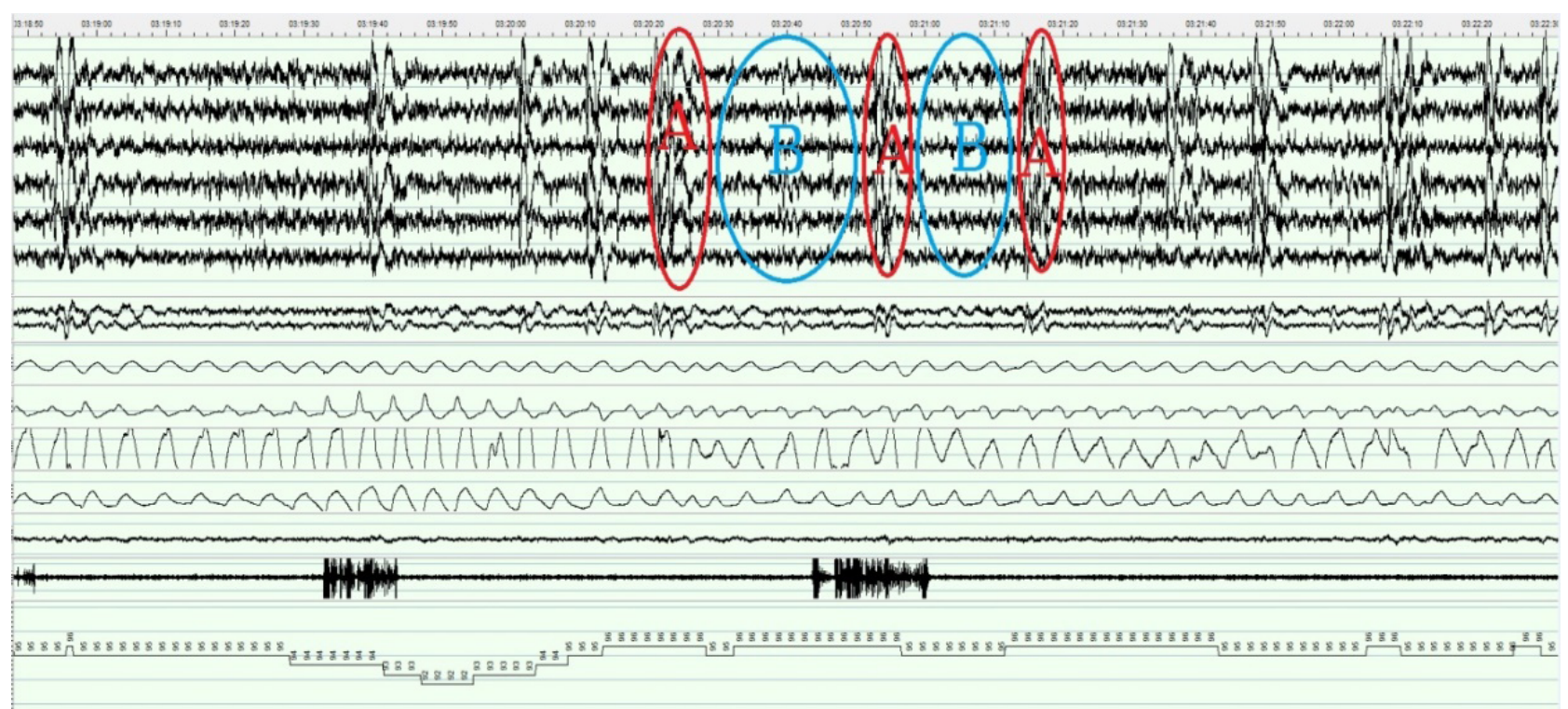


centage of lighter sleep, daytime sleepiness, daytime napping and the number of nocturnal awakenings increase with age, regardless of medication and comorbidities (5-8). However, some researchers argue that the effect of aging on REM sleep is unclear (9). Studies have examined the effect of age on CAP structure and have found that CAP rates and the ratio of CAP phases change depending on age and comorbidities (10).

Along with general deterioration in health among the elderly, there is an increased frequency of sleep disordered breathing, especially obstructive sleep apnea (OSA) (11). OSA is characterized by repetitive interruption of airflow during sleep, sleep fragmentation, snoring and day time sleepiness. The prevalence of OSA varies between $9 \%-38 \%$ in the general population and increases with age (12). A previous community-dwelling study reported a prevalence of sleep apnea1.7 times higher than average in subjects older than 60(13). Prevalence rates of severe OSA were $24 \%$ in the 65-95-year-old population (14).

Sleep apnea in the elderly worsens quality of life, existing diseases and such functions, as memory and learning, attention, motor and balance control. Sleep fragmentation, periodic nocturnal oxygen desaturation and increased sympathetic activation during sleep are major factors that change sleep structures, leading to daytime sleepiness and increasing the risk of cardio- and cerebrovascular events (15).

The diagnosis and severity of sleep apnea requires polysomnographic (PSG) investigation, including EEG, electro-oculography (EOG) and electromyography (EMG) as well as body position, cardiac, respiratory, audio and video monitoring during sleep. In addition to the usual scoring, this study employed a more advanced CAP scoring. Studies have shown that upper airway obstruction due to sleep apnea occurs in temporal connection with CAP. As a sensitive assessment, CAP involves EEG activity, muscle tone and autonomic responses even in the absence of apnea. Researchers believe that CAP analysis is a sensitive marker for detecting undercover sleep alterations that are not determinable by conventional scoring $(9,16,17)$.

So far, a lot of research on sleep architecture has been done in populations with sleep apnea and mainly focused on young and middle-aged adults. We were attracted to this topic to try to understand the potential relationship between aging and sleep breathing disorders by exploring such a valuable dimension as the CAP. The authors suggest that differences in sleep macro- and micropatterns that normally exist between elderly and middle-aged adults would persist even in the presence of severe apnea.

\section{MATERIAL AND METHODS}

This retrospective study was approved by the Ethical Committee of the Local University Faculty of Medicine (2020/01) and performed at our sleep center between May 2016 and January 2020. This study was conducted according to the principles expressed in the Declaration of Helsinki.

The inclusion criteria were male gender and diagnosis of severe obstructive sleep apnea. The exclusion criteria were previous diagnosis or treatment of sleep disorders, such as central sleep apnea, obesity-hypoventilation syndrome, restless leg syndrome, periodic limb movement disorders, insomnia or parasomnia and serious systemic disorders such as heart, kidney or liver failure, uncontrolled hypertension and diabetes mellitus. Patients using certain medications within the previous month, such as antihistamines, antidepressants, sedative-hypnotics and neuroleptics were excluded from the study.

Of the 680 patients who underwent PSG, 47 patients who met our criteria were included in the study. These 47 patients were divided into two groups according to their ages: 23 patients aged 65 and over were in the first group, and 24 patients between $35-45$ years were in the second group. The age, body mass index (BMI) and Epworth sleepiness 
scale (ESS) scores of both groups were recorded (18).

\section{Polysomnography}

PSG was performed for both groups with video monitoring. No hypnotic drugs were allowed before recording. The recordings were carried out using a computerized recording workstation (Embla $®$ RemLogic $^{T M}$ ).EEGs were recorded using bipolar montages(F3-M2, F4-M1, C3-M2, C4-M1, O1-M2 and O2-M1).The following parameters were recorded: body position, electrooculogram (EOG), electrocardiogram (ECG), electromyogram (EMG) of the submental space and both tibialis anterior muscles, oxygen saturation, nasal cannula, thermistor, snoring recorded by a microphone and respiratory efforts detected by inductance plethysmography.

EEG signals were subdivided into 30-s epochs and manually scored according to the American Academy of Sleep Medicine (AASM) Manual (19). Sleep scoring included stage W (wakefulness), NREM (stages N1, N2 andN3), REM, arousals, leg movements and respiratory measurements (apne- as/hypopneas and desaturation indexes). AHI was calculated as the count of apneasor hypopneas per hour of sleep. The oxygen desaturation index (ODI) was calculated as the number of desaturations (decrease of $\mathrm{SpO} 2 \geq 3 \%$ ) per hour of sleep.

From asleep macrostructure viewpoint, we calculated TST, SE, WASO, SL, REM sleep latency, arousal index and percentages of N1, N2, N3 and REM sleep.

These definitions are below:

Total sleep time: the time from sleep onset to the end of the final sleep epoch without time awake.

Sleep efficiency: the percentage ratio between total sleep time and time in bed.

Wake after sleep onset: the time spent awake in total sleep time.

Sleep latency: the interval between lights-off and the first appearance of a sleep stage.

REM sleep latency: the interval between lightsoff and the first REM sleep stage.

Arousal index: number of arousals per hour.

Figure 2. Cyclic alternating pattern $A 1, A 2$ and $A 3$ subtypes.

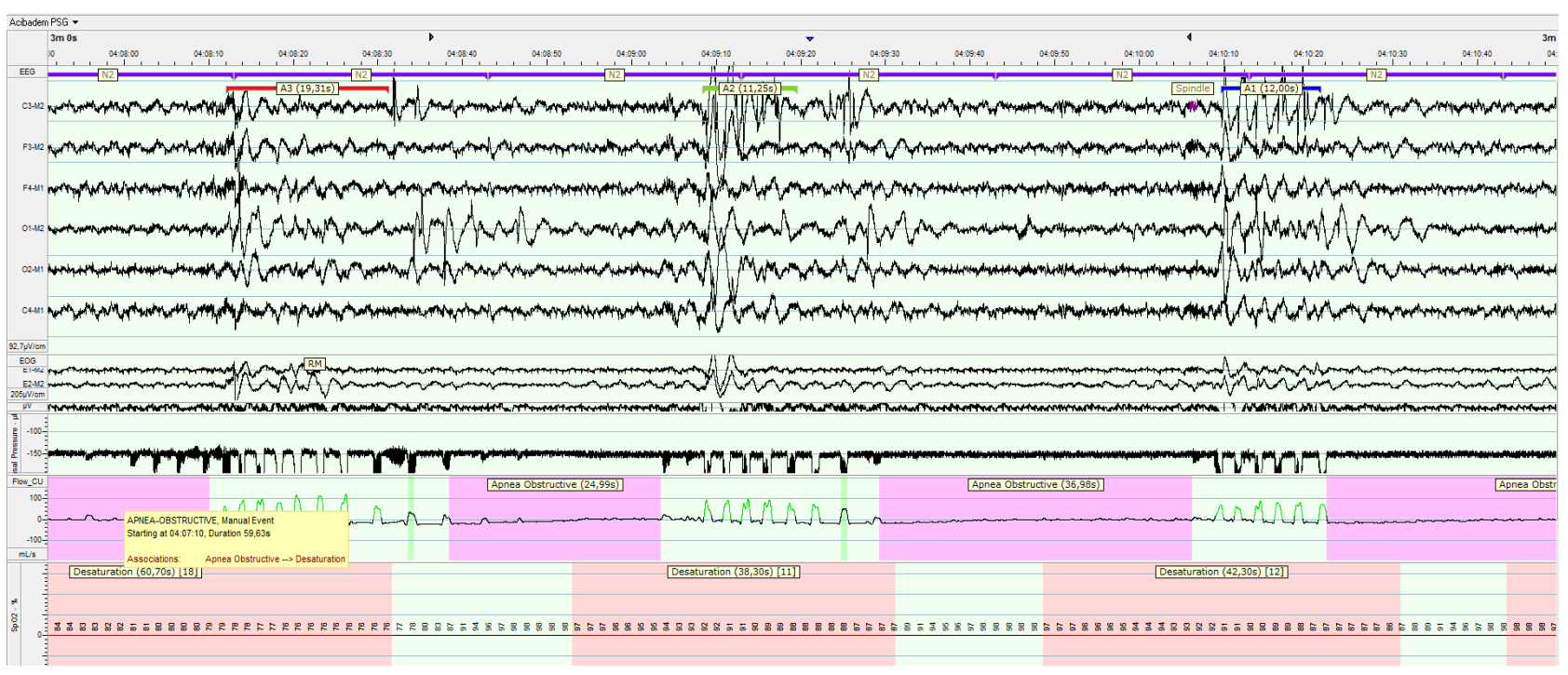


Figure 3. Cyclic alternating pattern A1 and A3 subtypes.

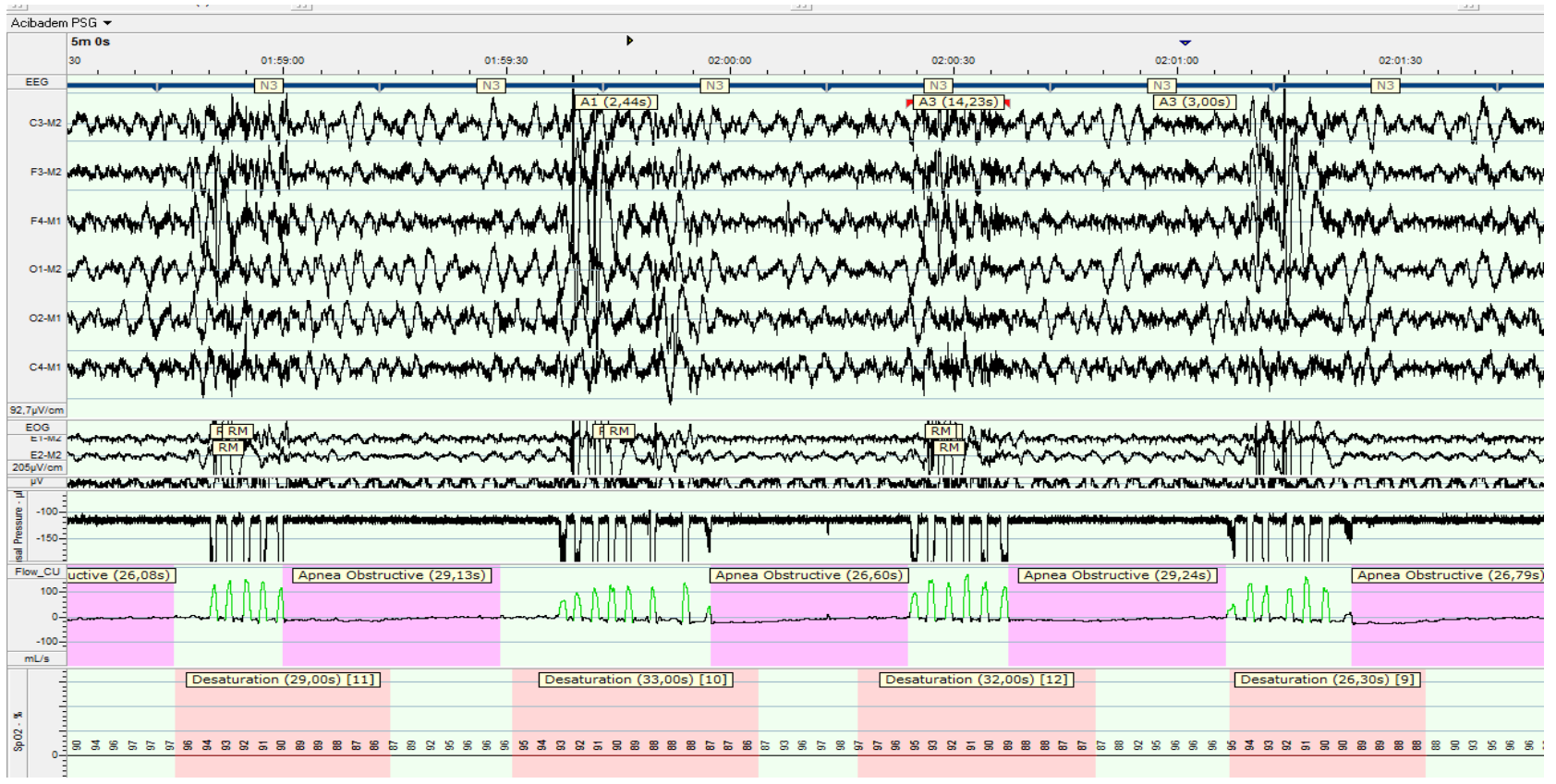

Figure 4. Cyclic alternating pattern $\mathrm{A} 1$ and $\mathrm{A} 2$ subtypes.

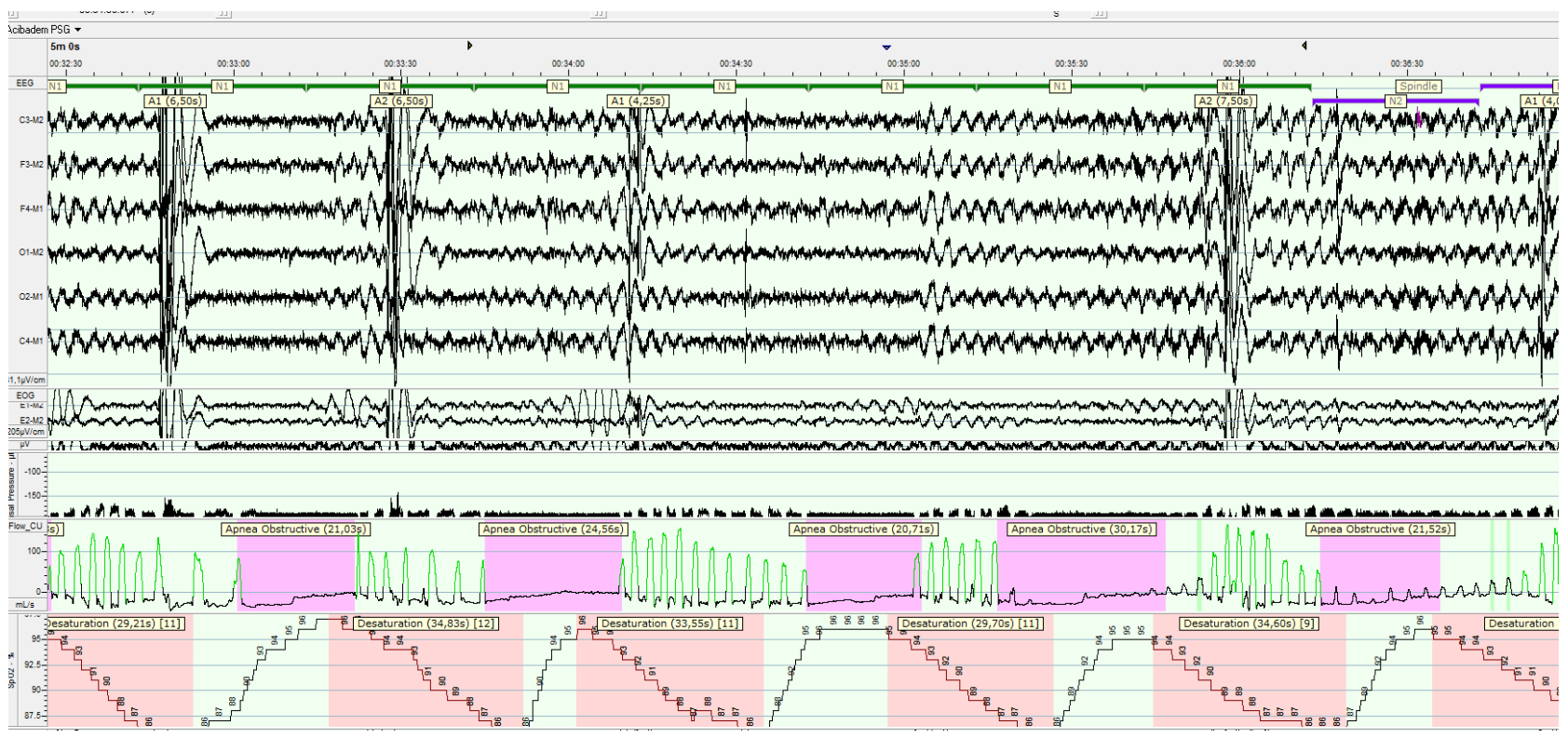


Percentage of N1, N2 N3 or REM sleep: percentage ratio of each sleep stage to total sleep time.

CAP scoring was annotated according to the conventional criteria (20). These are defined as follows:

A CAP sequence is composed of a succession of CAP cycles. Of the CAP cycles, $\geq 2$ were compound CAP sequences.

A CAP cycle is composed of a phase A (activation period) and phase $B$ (subsequent background period) (Figure 1).

Phase $A$ is divided into three subtypes: $A 1, A 2$ and $\mathrm{A} 3$.

Subtype $\mathbf{A} 1$ is composed of low-frequency activity, such as K-complexes and slow waves (Figures 2-4).

Subtype $\mathbf{A} 2$ is a transitional type between A1 and $A 3$ and is composed of mixture of slow and fast EEG activities (Figure 4).

Subtype $\mathbf{A} 3$ is represented by $>50 \%$ of high-frequency activities, such as EEG arousals and polyphasic bursts (Figure 2-3).

The following CAP parameters were calculated: CAP rate (percentage of CAP time to total NREM sleep time) and, separately, percentages of phase A1, A2 and A3 time to total NREM sleep time.

All recordings were carried out by automatic scoring with the subsequent control and manual corrections. They were then calculated for statistical analysis.

\section{Statistical Analysis}

SPSS (Statistical Package for Social Sciences) 15.0 for Windows was used for statistical analysis. A Chi-square test was used to compare qualitative data. The Kolmogorov-Smirnov test was used to determine whether the data were normally distributed. The nonparametric Mann-Whitney $U$ test was used for non-normal data, while the parametric Student t-test was used for normally distributed data.
Normally distributed variables were expressed as mean $\pm S D$, while skewed variables were expressed as median (interquartile range). Pearson and Spearman's correlation analyses were used to assess correlation between ESS and BMI scores and various sleep structure parameters. Values of $p<0.05$ were accepted as significant.

\section{RESULTS}

The average age of 23 male patients with severe OSA in the first group was $67.9 \pm 6.91$ years, while the group 2 was $41.0 \pm 4.40$ years. BMI was found to be significantly higher in group 1 patients (group 1 $\mathrm{BMI}=36.01$; group $2 \mathrm{BMI}=31.5 ; \mathrm{p}=0.011$ ). There was also a statistically significant difference between the groups in terms of ESS (group 1 mean ESS = 13.6, group 2 mean ESS $=8.7, p=0.001$ ). On the other hand, the groups showed no significant difference in ODI, which were $48.5 \pm 17.5$ and $52.8 \pm 20.3$ in the elderly and middle-aged groups, respectively. There were no statistically significant differences for all macro- and microstructural parameters (Table 1). Also, there was no significant correlation between $\mathrm{BMI}$ and ESS scores and sleep macro- and microparameters.

\section{DISCUSSION}

Based on this research, we have encountered unexpected results: data on the macrostructure of sleep in middle-aged apneic subjects statistically approached the data of elderly patients with the same disease, and these are also basically similar to the parameters of previous studies. In terms of microstructure, there were also no statistically significant differences between the two groups. Although the CAP rates in both groups from our study do not differ from each other or, on the whole, from the results of similar studies, our CAP subparameters were different from most previous works.

The authors did not find the literature a comparative study of sleep structure in severe apnea in different age categories. Many studies exploring the 
Table 1. Comparison of parameters of sleep macro- and microstructure in Group 1 and Group 2 . values of $p<0.05$ were accepted as significant.

\begin{tabular}{|c|c|c|c|}
\hline & Group 1 & Group 2 & $p$ \\
\hline TST (min) & $342.3 \pm 31.8$ & $335.1 \pm 31.0$ & 0.44 \\
\hline SE (\%) & $90.2 \pm 7.7$ & $88.1 \pm 9.2$ & 0.39 \\
\hline WASO (min) & $25.1 \pm 26.0$ & $28.9 \pm 33.2$ & 0.66 \\
\hline $\mathrm{SL}(\min )$ & $10.3 \pm 7.3$ & $14.1 \pm 8.5$ & 0.11 \\
\hline REM sleep latency (min) & 119.7(99.7) & $124.4(90.4)$ & 0.65 \\
\hline NREM N1 stage latency (min) & $15.4 \pm 8.7$ & $12.4 \pm 7.9$ & 0.23 \\
\hline NREM N2 stage latency (min) & $40.8 \pm 13.0$ & $40.7 \pm 15.0$ & 0.96 \\
\hline NREM N3 stage latency (min) & $33.8 \pm 20.2$ & $31.6 \pm 13.4$ & 0.66 \\
\hline N3 stage percentage (\%) & $11.42 \pm 9.91$ & $13.58 \pm 7.45$ & 0.39 \\
\hline REM sleep percentage (\%) & $12.4 \pm 11.6$ & $14.1 \pm 8.9$ & 0.56 \\
\hline Arousal index & $23.0 \pm 24.6$ & $14.7 \pm 14.4$ & 0.17 \\
\hline CAP rate (\%) & $35.5(18.1)$ & $38.2(20.9)$ & 0.58 \\
\hline Phase A1 percentage (\%) & $25.8(17.0)$ & $26.7(16.8)$ & 0.92 \\
\hline Phase A2 percentage (\%) & $5.6(6.0)$ & $5.6(3.9)$ & 0.96 \\
\hline Phase A3 percentage (\%) & $4.1(4.5)$ & $5.9(5.7)$ & 0.27 \\
\hline $\mathrm{AHI}$ & $48.2 \pm 17.0$ & $52.6 \pm 17.9$ & 0.39 \\
\hline
\end{tabular}

Data are presented as mean \pm SD or median (interquartile range)

Abbreviations: TST: total sleep time; SE: sleep efficiency; WASO: wake after sleep onset; SL: sleep latency, CAP: cyclic alternating pattern; REM: rapid eye movement sleep; NREM: non-rapid eye movement sleep; AHI: apnea-hypopnea index

effect of age on sleep architecture have been carried out among healthy and in mixed groups consisting of healthy subjects and those suffering from various diseases, including sleep apnea.

Age-related sleep structural changes were confirmed by two different meta-analyses $(5,21)$. Based on these and other previous investigations, TST and SE were significantly reduced in elderly subjects. On the other hand, there are contradictory reports of whether SL varies with age. Most studies have shown a positive association between SL and increasing age, particularly in studies that compared very young and elderly subjects. Our results showed statistically similar TST, SE and SL values in both groups, regardless of age.

Studies included participants with OSA have shown no significant correlation between age and percentage of N1 and N2 sleep (5,21). Similarly, in the present study, N1 and N2 percentage did not change between the different age groups.

In contrast, the percentage of SWS and REM sleep decreases with age in adults $(5,6)$. The exclusion or inclusion of participants with OSA did not change the outcomes in all cases. Other authors have reported a small increase in the proportion of REM between ages 75 and 85 (21). The percentage and latency of REM sleep are close to values of a previous study whose participants were patients with severe OSA and an average age of 38.58 years (22). However, the percentage of slow sleep in our patients is significantly higher than in patients in that study.

Difficulty of sleep maintenance, which presents 
as a longer duration of WASO and increased arousal index, tends to decrease up to the age of sixty and remains mostly unchanged after this age, regardless of the presence of sleep apnea (6). Arousal index values are similar to the data of previous studies, but WASO values are significantly different $(17,22)$. This variability can be explained by the duration of polysomnographic monitoring and the patient's time leaving the device.

Usually, the CAP rate is correlated with age in healthy persons. Since the CAP rate is the measure of arousability, higher CAP rates are associated with poorer quality of sleep, the CAP rate shows a bimodal distribution along the normal lifespan with two peaks during adolescence and senescence, respectively. It reflects the development and subsequent decline of physiological processes (10).

Many previous studies have confirmed that untreated patients with OSA present an exaggerated increase of CAP rates. For example, a recent study that examined CAP rates in participants aged between 40 and 70 suggested a significant increase in CAP rates among OSA patients $(50.01 \pm 10.6)$ versus control subjects (19.3 \pm 7.9 ) (15). Another study reported that untreated middle-aged patients with OSA presented extremely increased CAP rates(3). The clinical review of Parrino et al. indicated that CAP rates in healthy middle-aged and elderly subjects were approximately $37.5 \%$ and $55.3 \%$, respectively (4). In our study, these rates were $35.3 \%$ in middle-aged participants and $39.1 \%$ in elderly OSA patients. CAP rates from the literature review and the present study are similar in middle age, although these compare healthy (in review) and OSA (in our study) patients. On the other hand, our elderly patients with OSA had lower CAP rates than elderly populations in the review. This can be explained by the fact that there were data used from a mixed population (healthy and with various comorbidities) in the review, while the present study chose only patients with severe OSA.

In the present study, both groups showed statistically similar phase A1, A2 and A3 percentages, but different counts of phase A subtypes (more A1 phases than $A 2$ and $A 3$ ). As known, phase $A 1$ is associated with restorative processes in healthies; and decreased arousability in some disorders (4). Our data were different from previous studies, in which the percentage of $\mathrm{A} 1$ phases was significantly lower than those of phases $A 2$ and $A 3(4,22)$. Full parallelism between our study and previous studies is impossible due to the different ages, comorbidities, activity levels, medications and cognitive profiles, which could determine the difference in phase A subtypes.

The authors would like to note that this work was carried out in high altitude conditions $(1100 \mathrm{~m}$ above sea level) and most patients were from nearby settlements located at a high altitude. The effect of high altitude on the microstructure of sleep is still unexplored.

There may also be other methodological reasons that led to the above results. The different EEG frequencies, particularly components of CAP, are not equally distributed over the scalp. The phase A1 components show a clear prevalence and are best recorded over the anterior frontal regions for the $0.25-2.5 \mathrm{~Hz}$ band; phase A3 components register best over the parietooccipital areas for the 7-12 Hz band (4). In our study, the CAP scoring was made from all recording electrodes. These EEG montages were mostly located in the frontocentral region. Since the $A 3$ subtype mostly occurs in the parietooccipital region, the existing $01-\mathrm{M} 2$ and O2-M1 montages may not be sufficient for detecting A3 subtype. Another explanation could be that slow rhythms (the main part of phase A1) and autonomic functions may have a reciprocal interaction in senescence. Autonomic reactions become softer and slower with advanced age. OSA patients have also observed termination of respiratory events with a predominant increase of delta power, which is a part of phase A1(4). Finally, the increase of slow oscillations and reduction of phases $A 2$ and $A 3$ can be explained by deterioration of the arousal system in elderly populations. 
One major limitation of the present study is the absence of a healthy control age-matched group and the lack in the literature of normative data on structures of sleep for elderly healthy people. These make it impossible to compare healthy and OSA patients. Other limitations of the present study were the small number of participants and inclusion of only male patients. We chose to work only with male participants because elderly men experience much greater impairment in NREM sleep than agematched women (7).

All these considerations indicate the need for future investigation of geriatric sleep in different health conditions. External and internal arousal-re-

\section{REFERENCES}

1. Terzano MG, Mancia D, Salati MR, Costani G, Decembrino A, Parrino L. The cyclic alternating pattern as a physiologic component of normal NREM sleep. Sleep1985;8(2):137-45. (PMID:4012156)

2. Muzet A. Alteration of sleep microstructure in psychiatric disorders.Dialogues Clin Neurosci. 2005;7(4):315-21. (PMID:16416707)

3. Parrino L, Smerieri A, Boselli M, Spaggiari MC, Terzano MG. Sleep reactivity during acute nasal CPAP in obstructive sleep apnea syndrome. Neurology 2000;54(8):1633-40. (PMID:10762505)

4. Parrino L, Ferri R, Bruni $O$, Terzano MG. Cyclic alternating pattern (CAP): the marker of sleep instability. Sleep Med Rev 2012;16(1):27-45. (PMID:21616693)

5. Ohayon MM, Carskadon MA, Guilleminault C, Vitiello MV. Meta-analysis of quantitative sleep parameters from childhood to old age in healthy individuals: developing normative sleep values across the human lifespan.Sleep2004;27(7):1255-73. (PMID:15586779)

6. Li J, Vitiello MV, Gooneratne NS. Sleep in normal aging. Sleep Med Clin 2018;13(1):1-11 (PMID:29412976)

7. Mander BA, Winer JR, Walker MP. Sleep and human aging. Neuron 2017;94(1):19-36.(PMID:28384471)

8. Crowley K. Sleep and sleep disorders in older adults. Neuropsychol Rev2011;21(1):41-53.( PMID:21225347)

9. Schwarz JFA, Åkerstedt T, Lindberg E, Gruber G, Fischer $\mathrm{H}$, Theorell-Haglöw J. Age affects sleep lated conditions must be taken into account, such as homeostatic, neuropsychiatric and autonomic system status, diurnal vigilance, presence of any pain, comorbidities, drug application, relative inconvenience of sleep in a laboratory, ambient temperature and noise. It is obvious that all data need careful analysis in light of internal and environmental factors. We believe that a future abundance of studies on this topic will answer these questions about daily clinical applications of microstructural analyses on the diagnosis of diseases and effect of treatment.

Conflicts of interest: The authors declare that they have no conflict of interest.

microstructure more than sleep macro structure. J Sleep Res 2017;26(3):277-287. (PMID:28093830)

10. Parrino L, Boselli M, Spaggiari MC, Smerieri A, Terzano MG. Cyclic alternating pattern (CAP) in normal sleep: polysomnographic parameters in different age groups. Electroencephalogr Clin Neurophysiol. 1998;107(6):439-50. (PMID:9922091)

11. YoungT, PeppardE, Gottlieb DJ. Epidemiology of obstructive sleep apnea: a population health perspective. Am J RespirCritCareMed2002;165(9):1217-39. (PMID:11991871)

12. Senaratna CV, Perret JL, Lodge CJ et al. Prevalence of obstructive sleep apnea in the general population: A systematic review. Sleep Med Rev 2017;34:7081. (PMID:27568340)

13. Young T, Shahar E, Nieto J et al. Predictors of sleep-disordered breathing in community-dwelling adults: the Sleep Heart Health Study. Arch Intern Med 2002;162(8):893-900. (PMID:11966340)

14. Ancoli-Israel S Kripke DF, Klauber MR, Mason WJ, Fell $R$, Kaplan $O$. Sleep-disordered breathing in community-dwelling elderly. Sleep 1991;14(6):486495. (PMID:1798880)

15. Karimzadeh F, Nami M, Boostani R. Sleep microstructure dynamics and neurocognitive performance in obstructive sleep apnea syndrome patients. J Integr Neurosci 2017;16(2):127-142. (PMID:28891505) 
16. Parrino L, Grassi A, Milioli G. Cyclic alternating pattern in polysomnography: what is it and what does it mean? Curr Opin Pulm Med. 2014;20(6):533-541. (PMID:25188718)

17. Priano L, Bigoni M, Albani G, et al. Sleep microstructure in Parkinson's disease: cycling alternating pattern (CAP) as a sensitive marker of early NREM sleep instability. Sleep Med 2019; 61:57-62. (PMID:31307885)

18. Izci B, Ardic S, Firat H, Sahin A, Altinors M, Karacan I. Reliability and validity studies of the Turkish version of the Epworth Sleepiness Scale. Sleep Breath 2008; 12:161-168. (PMID:17922157)

19. Iber C, Ancoli-Israel S, Chesson AL, Jr., Quan SF. The AASM manual for the scoring of sleep and associated events: rules, terminology and technical spec- ifications. In: Iber C et al (Eds). American Academy of Sleep Medicine.1st edition, Westchester, IL USA 2007, pp 3-47.

20. Terzano MG, Parrino L, Smerieri A, et al. Atlas, rules, and recording techniques for the scoring of cyclic alternating pattern (CAP) in human sleep. SleepMed 2002;3(2):187-199. (PMID:14592244)

21. Floyd JA, Medler SM, Ager JW, Janisse JJ. Age-related changes in initiation and maintenance of sleep: a meta-analysis. Res Nurs Health 2000;23(2):106-117. (PMID:10782869)

22. Li N, Wang J, Wang D, et al. Correlation of sleep microstructure with daytime sleepiness and cognitive function in young and middle-aged adults with obstructive sleep apnea syndrome. Eur Arch Otorhinolaryngol 2019;276(12):3525-3532. (PMID:31263979 ) 